Pacific Journal of Mathematics

COBORDISM CLASSES OF FIBER BUNDLES 


\title{
COBORDISM CLASSES OF FIBER BUNDLES
}

\author{
DANIEL F. X. O'REILLY
}

This paper treats the problem of determining which unoriented cobordism classes have a representative which is the total space of a fiber bundle over a sphere. We are looking for a necessary and sufficient condition for a closed, compact, differentiable manifold to be cobordant to the total space of a fiber bundle over $S^{k}$.

Our results on bundles over $S^{4}$ and $S^{8}$ extend the results of P. E. Conner [3, 4], E. E. Floyd [4], R. O. Burdick [2], W. D. Neumann [6], R. L. W. Brown [1] and R. E. Stong [7].

The following definition will facilitate the discussion.

DEFINITION 0.1. If $\alpha$ represents an unoriented cobordism class, we say that $\alpha$ fibers over $S^{k}$ if $\alpha$ contains a representative which is the total space of a fiber bundle over $S^{k}$.

It has been shown (see [4] and [1]) that [ $\left.M^{n}\right]$ fibers over $S^{1}$ if and only if $\left\langle w_{n},\left[M^{n}\right]\right\rangle$, which we will abbreviate by $w_{n}\left[M^{n}\right]$, is zero, and $\left[M^{n}\right]$ fibers over $S^{2}$ if and only if

$$
\begin{cases}w_{n}\left[M^{n}\right]=0 & \text { if } n \text { is even } \\ w_{n-2} w_{2}\left[M^{n}\right]=0 & \text { if } n \text { is odd . }\end{cases}
$$

The last condition is also sufficient for the cobordism class of a manifold to fiber over any particular $N^{2}$, since Stong has shown that if $\left[M^{n}\right]$ fibers over $S^{k}$, then it fibers over any manifold of dimension less than or equal to $k$ (see [7]).

Our main results are as follows.

THEOREM I. There are generators of $\mathfrak{\Omega}_{*}$ which fiber over $S^{4}$ in all dimensions greater than or equal to 8 except 11 and, of course, those of the form $2^{j}-1$.

CoRollary I. We can choose generators of $\Re_{*}=Z_{2}\left[x_{i} \mid i \neq 2^{j}-1\right]$ so that an element of either one of the following subalgebras will fiber over $S^{4}$ if and only if the Stiefel-Whitney numbers associated with $w_{n}$ and $w_{n-2} w_{2}$ are both zero. The subalgebras are:

$$
\begin{aligned}
& I=Z_{2}\left[x_{i} \mid i \neq 5,6,11 \text { or } 2^{j}-1\right] \\
& J=Z_{2}\left[x_{i} \mid i \neq 4,5,11 \text { or } 2^{j}-1\right] .
\end{aligned}
$$

THEOREM II. No indecomposable 11-dimensional class fibers 
over $S^{4}$. Also, the cobordism classes of $x_{4} x_{6}$ and $x_{5}^{2}$ (where $x_{4}=\left[P_{2}^{4}\right]$ and $x_{6}=\left[P_{2}^{6}\right]$ are classes which fiber over $S^{2}$ (see [1]) and $x_{5}$ is the indecomposable 5-dimensional class) do not fiber over $S^{4}$. (This result is a composite of Propositions 4.2, 5.1 and 5.2.)

Certain remarks should be made concerning these results. Theorem I leaves several open questions. For example it is not known whether classes like $x_{11} x_{2}$ or $x_{5}^{3}$ fiber over $S^{4}$, although it is conjectured that they do not. Theorem II can be regarded as somewhat surprising, since it is known that Stiefel-Whitney numbers involving high dimensional $(>6)$ Stiefel-Whitney classes for any indecomposable $x_{11}$ are zero, and just such a condition is sufficient for a cobordism class to fiber over $S^{2}$ or $S^{1}$. Although it is not very surprising that $x_{5}^{2}$ fails to fiber over $S^{4}$ (since $x_{5}$ does not fiber over $S^{2}$ ), it is rather surprising that $x_{4} x_{6}$ fails to fiber over $S^{4}$. For R. L. W. Brown has shown that the cobordism class of a product of manifolds which fiber over $S^{1}$ must fiber over $S^{2}$, and that if $M$ fibers over $S^{2}$, then $[M \times M]$ fibers over $S^{4}[1]$. So it would have been natural to conjecture that if $M$ and $N$ both fiber over $S^{2}$, then $[M \times N]$ fibers over $S^{4}$.

Several other results are worthy of note. In $\S 3$ we construct a 9-dimensional manifold which fibers over $S^{4}$ and is cobordant to the 9-dimensional Dold generator. In $\$ 5$ certain results concerning the fibering of 15-dimensional classes are proved using the results of a computer study in that dimension. Finally, generators of unoriented cobordism which fiber over $S^{8}$ are given for even dimensions greater than or equal to 16 and odd dimensions greater than or equal to 25 .

1. A necessary condition. As is well known, if $M^{n}$ is the total space of a fiber bundle over $S^{k}$ with fiber, $F$, then we can write the tangent bundle to $M^{n}$ as follows:

$$
\tau M^{n}=p^{*} \tau S \oplus \bar{\tau} F^{n-k}
$$

where $\bar{\tau} F^{n-k}$ is a bundle over $M^{n}$ such that $i^{*} \bar{\tau} F^{n-k}=\tau F^{n-k}$ for each fiber. But $W\left(S^{k}\right)=1$; so by the Whitney product formula,

$$
W\left(M^{n}\right)=W\left(\bar{\tau} F^{n-k}\right),
$$

which implies $w_{i}\left(M^{n}\right)=0$ if $i>n-k$. From this we can conclude that Stiefel-Whitney numbers associated with monomials divisible by $w_{n}, w_{n-1}, \cdots$, and $w_{n-k+1}$ must be zero. R. L. W. Brown [1] has shown that if $n$ is odd and $M^{n}$ fibers over $S^{2}$, then $w_{n-2}$ also vanishes. This result can be generalized for all even dimensional spheres. 
Proposition 1.1. If $M^{2 n+1}$ is cobordant to $N^{2 n+1}$ which fibers over $S^{2 k}$, then Stiefel-Whitney numbers associated with monomials divisible by $w_{2 n+1-2 k}$ must be zero.

The proof is almost identical to the one given by Brown for $S^{2}$ and will be omitted. As a consequence of 1.1 , if $\left[M^{n}\right]$ fibers over $S^{2 k}$, then Stiefel-Whitney numbers associated with monomials divisible by

$$
\begin{cases}w_{n}, w_{n-1}, \cdots, w_{n-2 k+1} & \text { for } n \text { even } \\ w_{n}, w_{n-1}, \cdots, w_{n-2 k} & \text { for } n \text { odd }\end{cases}
$$

are zero.

2. Generators for unoriented cobordism which fiber over $S^{4}$. In this section we will prove the first of the two main technical results needed for Theorem I. First we recall the manifolds introduced by Conner and Floyd and R. L. W. Brown (see [4] and [1]):

$$
P_{4}^{m+4}=R P\left(H_{4} \oplus(m-3) R\right)
$$

and

$$
P_{4}(m, n)=R P\left(H_{4} \times \tau P^{n} \oplus(m-3) R\right),
$$

where $H_{4}$ is the canonical twisted quaternionic line bundle over $\boldsymbol{Q} P^{1}=S^{4}$, considered as a real vector bundle. Notice that $P_{4}(m, n)$ has dimension $m+2 n+4$.

Lemma 2.1 [1, Propositions 3.2 and 3.4]. If $m$ is even, then $\left[P_{4}^{m+4}\right]$ is indecomposable, and $\left[P_{4}(m, n)\right]$ is indecomposable if $n$ is even and

$$
\left(\begin{array}{c}
m+n+3 \\
n
\end{array}\right) \equiv 1 \bmod 2
$$

Proposition 2.2. There exist generators of $\mathfrak{N}_{*}$ which fibers over $S^{4}$ in even dimensions $\geqq 8$ and odd dimensions $\geqq 13$.

Proof. First, it is easy to see that even dimensional generators which fiber over $S^{4}$ are taken care of by:

$$
\left\{\left[P_{4}^{m+4}\right] \mid m \in 2 Z \text { and } m \leqq 4\right\} \text {. }
$$

Now suppose $i$ is odd and not of the form $2^{j}-1$. Let

$$
i=2^{p}(2 q+1)-1,
$$


where $p, q>0$. The unoriented cobordism class of

$$
P_{4}\left(2^{p}-5,2^{p} q\right)
$$

is indecomposable for $p>2$, and therefore gives a generator for all odd dimensions except those of the form $4 q+1$ and $8 q+3$.

For dimensions of the form $4 q+1$, we use the manifolds,

$$
P_{4}(4 q-7,2) \text {. }
$$

These manifolds clearly have the proper dimension and satisfy both conditions of Lemma 2.1. However, it is clear that $q$ must be greater than 2 , for otherwise the definition of $P_{4}(4 q-7,2)$ would be meaningless. In other words, we have "missed" dimensions 5 and 9.

For dimensions of the form $8 q+3$, we use the cobordism classes of the manifolds,

$$
P_{4}(8 q-9,4) \text {. }
$$

The obvious restriction this time is $q>1$, so that we miss dimension 11. Altogether then, we have indecomposable generators which fiber over $S^{4}$ in all dimensions $\geqq 8$, which are not of the form $2^{j}-1$, except 9 and 11. The next section is devoted to a construction of a 9-dimensional manifold over $S^{4}$ whose cobordism class is indecomposable, thus completing the proof of Theorem I.

Proof of Corollary I. Let $x_{4}=\left[P_{2}^{\dagger}\right]$ and $x_{6}=\left[P_{2}^{6}\right]$ and thereafter choose $x_{i}$ so that it fibers over $S^{4}$. We will prove the result for $I$ only, since the proof is similar for $J$. Now suppose $\left[M^{n}\right]$ is any cobordism class belonging to the subalgebra $I$, for which $w_{n}\left[M^{n}\right]=$ $w_{n-2} w_{2}\left[M^{n}\right]=0$. Suppose furthermore that $n=2 k$ (for if $n$ odd, it fibers trivially). Then we can write

$$
\left[M^{n}\right]=\left[N^{n}\right]+r x_{2}^{k}+s x_{2}^{k-2} x_{4},
$$

where $\left[N^{n}\right]$ fibers over $S^{4}$ and $r, s \in Z_{2}$. Applying $w_{n}$ to both sides forces $r=0$, and then applying $w_{n-2} w_{2}$ to both sides forces $s=0$.

3. A 9-dimensional generator which fibers over $S^{4}$. Since bundles over $S^{4}$ are classified by $\pi_{3}(G)$, where $G$ is the structure group, we must construct a map from $S^{3}$ into Diff $\left(F^{5}\right)$ where $F^{5}$, the fiber, is defined by:

$$
F=\boldsymbol{R} \times \boldsymbol{C} P^{2} /(r, z) \sim(s, \bar{z}) \text { if } r=s+1,
$$

(where, if $z=\left(z_{1}, z_{2}, z_{3}\right)$, then $\bar{z}=\left(\bar{z}_{1}, \bar{z}_{2}, \bar{z}_{3}\right)$ ). If $\alpha \in S^{3}$, we let

$$
\alpha(t, z)=\left(t, \theta_{t}(\alpha) \cdot z\right)
$$


define a left action on $F$, where $\theta_{t}(\alpha) \in G L(2, C)$, and its action on $z$ is defined as follows. Let $\alpha=A+B j$ where $A$ and $B$ are complex. Then

$$
\theta_{t}(\alpha):=\left(\begin{array}{cc}
\cos \frac{\pi t}{2} & \sin \frac{\pi t}{2} \\
-\sin \frac{\pi t}{2} \cos \frac{\pi t}{2}
\end{array}\right)\left(\begin{array}{rr}
A & -B \\
\bar{B} & \bar{A}
\end{array}\right)\left(\begin{array}{rr}
\cos \frac{\pi t}{2} & -\sin \frac{\pi t}{2} \\
\sin \frac{\pi t}{2} & \cos \frac{\pi t}{2}
\end{array}\right)
$$

and $\theta_{t}(\alpha) \cdot\left(z_{1}, z_{2}, z_{3}\right)=T^{-1}\left(\theta_{t}(\alpha)\left(\begin{array}{l}z_{1} \\ z_{2}\end{array}\right), z_{3}\right)$, where $T\left(z_{1}, z_{2}, z_{3}\right)=\left(\left(\begin{array}{l}z_{1} \\ z_{2}\end{array}\right), z_{3}\right)$ defines the obvious bijection between $C P^{2}$ and $C^{2} \times C-\{0\}$ with the proper equivalence relation. It is straightforward to check that this action is well-defined. Finally we let $M^{9}$ be the total space of the bundle so constructed.

It now remains to determine the Stiefel-Whitney numbers of $M^{9}$. First, we claim that $H^{*}\left(M^{9}\right)=E\left(e_{1}\right) \otimes P\left(e_{2}\right) \otimes E\left(e_{4}\right) / e_{2}^{3}=e_{2} e_{4}$, where $E\left(e_{k}\right)$ denotes an exterior algebra on a $k$-dimensional generator and $P\left(e_{k}\right)$ denotes a polynomial algebra on a $k$-dimensional generator. This follows from the associated Serre Spectral Sequence and the fact that $\boldsymbol{C P}\left(H_{4} \oplus C\right)$ is a subbundle. For $H^{*}(\boldsymbol{C P}(\xi))$ is determined by

$$
\Sigma(-1)^{k+1-j} p^{*}\left(c_{j}\right) t^{k+1-j}=0
$$

(where $\xi$ is any complex $(k+1)$-plane bundle, $t$ is the characteristic class of the canonical line bundle $\eta \rightarrow C P(\xi)$, and $c_{j}$ is the $j^{\text {th }}$ Chern class), and in this case

$$
e_{2}^{3}+p^{*}\left(c_{2}\right) e_{2}=0 \text {. }
$$

But $p^{*}\left(c_{2}\right)=e_{4}$, since $c_{2}$ is the generator of $H^{4}\left(S^{4} ; Z\right)$. To determine the $W u$ classes, it suffices to note that $S q^{4}\left(e_{1} e_{4}\right)=0$ and $S q^{4}\left(e_{1} e_{2}^{2}\right)=$ $e_{1} e_{2}^{4}=e_{1} e_{2}^{2} e_{4}$, and so $v_{4}=e_{4} ; S q^{3} e_{2}^{3}=0$ and $S q^{3} e_{2} e_{4}=0$, so $v_{3}=0$; and finally, $S q^{2} e_{1} e_{2}^{3}=e_{1} e_{2}^{2} e_{4}$, so $v_{2}=e_{2}$. Since the fiber is oriented, $w_{1}=v_{1}=0$. Now, according to $\mathrm{Wu}$ 's formula, $W=S q V$, we conclude: $w_{1}=0, w_{2}=e_{2}, w_{3}=e_{1} e_{2}$ and $w_{4}=e_{4}+e_{2}^{2}$. It is clear that the only nonzero Stiefel-Whitney number of $M^{9}$ is $w_{3} w_{2}^{3}\left[M^{9}\right]$ which implies that $M^{9}$ belongs to the same cobordism class as the Dold generator, $P(1,4)$. Thus a nine dimensional generator of $\mathfrak{N}_{*}$ which fibers over $S^{4}$ has been found.

4. Non-existence of a generator over $S^{4}$ for dimension 11. In this section we will prove that there are no indecomposable 11dimensional classes which fiber over $S^{4}$. The proof rests on the fact that, if $M^{11}$ is the total space of a fiber bundle over $S^{4}$, then $v_{4}\left(M^{11}\right)$ 
is the pull-back of the generator of $H^{4}\left(S^{4}\right)$. First, we need the following well-known lemma.

LEMMA 4.1. If $n=2^{i}$, then $w_{n}$ can be expressed as $v_{n}+($ terms involving products of lower dimensional Stiefel-Whitney classes). In particular, it is easy to show,

$$
w_{4}=v_{4}+w_{3} w_{1}+w_{2}^{2}+w_{1}^{4} .
$$

Proposition 4.2. There does not exist an indecomposable 11dimensional cobordism class which fibers over $S^{4}$.

Proof. Suppose $F^{7} \stackrel{i}{\rightarrow} M^{11} \stackrel{p}{\rightarrow} S^{4}$ is a fiber bundle and that $\left[M^{11}\right]$ is indecomposable. First, we claim $v_{4}(M)=p^{*}(x)$, where $x$ is the generator of $H^{4}\left(S^{4}\right)$. For let $v_{4}=r_{1} p^{*}(x)+r_{2} y$, where $r_{i} \in Z_{2}$ and let $i^{*}(y) \in H^{4}(F)$. If $r_{2} y \neq 0$, then $v_{4}(F)=r_{2} i^{*}(y) \neq 0$, which is nonsense since $F$ is only 7-dimensional. Hence, $r_{2} y=0$. (Note that $r_{1}$ cannot also be zero because $v_{4}$ is nonzero.)

Now, since $\left[M^{11}\right]$ is assumed indecomposable, $s_{11}[M] \neq 0$, but $s_{11}[M]=S q^{4} s_{7}[M]=v_{4} s_{7}[M]=\left\langle p^{*}(x) \cup s_{7},[M]\right\rangle=\left\langle s_{7}, p^{*}(x) \cap[M]\right\rangle=$ $\left\langle s_{7}, i_{*}[F]\right\rangle=\left\langle i^{*}\left(s_{7}\right),[F]\right\rangle=s_{7}[F]=0$, since all 7-dimensional classes are decomposable.

5. Investigations in the $10^{\text {th }}$ and $15^{\text {th }}$ dimension. It is in dimension 10 that some of the most appealing patterns first break down. For $4 \leqq n<10$, it is true that $\left[M^{n}\right]$ fibers over $S^{4}$ if and only if Stiefel-Whitney numbers involving monomials divisible by $w_{n}, w_{n-1}, w_{n-2}$ and $w_{n-3}$ are all zero. However, there is a problem when we consider $x_{5}^{2}$. It is true that Stiefel-Whitney numbers involving $w_{10}, w_{9}, w_{8}$, and $w_{7}$ are all zero, and therefore, applying a theorem of Strong (see [7, 7.2]), one can conclude that $x_{5}^{2}$ fibers over $\left(S^{1}\right)^{4}$. But we also have the following.

Proposition 5.1. The cobordism class, $x_{5}^{2}$, does not fiber over $S^{4}$.

Proof. Suppose $F^{6} \rightarrow M^{10} \rightarrow S^{4}$ is a fiber bundle and that $M$ is cobordant to the square of the 5-dimensional generator. Recall that according to the well-known results of C. T. C. Wall and J. Milnor, Stiefel-Whitney numbers divisible by odd $w_{i}$ are zero, so $w_{5} w_{4} w_{1}[M]=0$. But also, $w_{5} w_{4} w_{1}[M]=w_{5}\left(v_{4}+w_{3} w_{1}+w_{2}^{2}+w_{1}^{4}\right) w_{1}[M]=$ $w_{5} v_{4} w_{1}[M]=w_{5} w_{1}[F]$, as in the proof to 4.1 , so we conclude that $w_{5} w_{1}[F]=0$.

However, $w_{6} w_{4}[M]=w_{3} w_{2}\left[x_{5}\right]=1$, and again replacing $w_{4}$ by $v_{4}+w_{2}^{2}+w_{3} w_{1}+w_{1}^{4}$, we obtain 


$$
w_{6}[F]+w_{6} w_{2}^{2}[M]+w_{6} w_{3} w_{1}[M]+w_{6} w_{1}^{4}[M]=1 .
$$

The last two terms on the left must vanish, and $w_{6} w_{2}^{2}[M]=w_{3} w_{1}^{2}\left[x_{5}\right]$ is also zero. Hence $w_{6}[F]=1$. But this is absurd since

$$
w_{6}[F]=S q^{3} v_{3}[F]=S q^{1} S q^{2} v_{3}[F]=w_{1}\left(S q^{2} v_{3}\right)[F]=w_{1} w_{5}[F] .
$$

Proposition 5.2. The cobordism class, $x_{6} x_{4}$ (where $x_{6}=\left[P_{2}^{6}\right]$ and $\left.x_{4}=\left[P_{2}^{4}\right]\right)$, does not fiber over $S^{4}$.

Proof. Suppose a representative, $M$, of $x_{6} x_{4}$ fibers over $S^{4}$ with fiber $F$. Direct calculation shows that $w_{6} w_{4}[M]=1$, from which it follows as before that $w_{6}[F]=1$. However $w_{5} w_{1}[F]=w_{5} w_{1} v_{4}[M]=$ $w_{5} w_{4} w_{1}[M]+w_{5} w_{3} w_{1}^{2}[M]+w_{5} w_{2}^{2} w_{1}[M]+w_{5} w_{1}^{5}[M]=0$, which is again a contradiction.

Unfortunately, there is still an unanswered question in dimension 10. It is not known whether $x_{5}^{2}+x_{4} x_{6}$ fibers, although it is easy using the above techniques, to prove the following interesting result.

Proposition 5.3. If $x_{5}^{2}+x_{4} x_{6}$ admits a representative which fibers over $S^{4}$ with fiber $F$, then $F$ is null cobordant.

The question of which cobordism classes fiber over $S^{4}$ becomes especially complicated in the $15^{\text {th }}$ dimension. In particular, it would be nice to know whether $x_{5}^{3}, x_{11} x_{2}^{2}$, or $x_{11} x_{4}$ fiber-for any choice of $x_{11}$ and $x_{4}$. It seems reasonable to conjecture that $x_{5}^{3}$ and $x_{11} x_{2}^{2}$ do not fiber over $S^{4}$, but the techniques used earlier are useless here, since $v_{4}(F)$ need not be zero.

However, there is at least one method for getting some additional information. We can construct other manifolds which do fiber over $S^{4}$ and then express them in terms of a chosen set of generators. For example, $P_{4}(3,4)=R P\left(H_{4} \times \tau P^{4}\right)$ is a 15-dimensional manifold which fibers over $S^{4}$. An IBM 360/67 was used to compute the Stiefel-Whitney numbers for $P_{4}(3,4)$ and basis of $\mathfrak{R}_{15}$. The outcome was as follows:

$$
\begin{aligned}
{\left[P_{4}(3,4)\right]=x_{5}^{3} } & +x_{10} x_{5}+x_{8} x_{5} x_{2}+x_{9} x_{6}+x_{9} x_{4} x_{2} \\
& +x_{6} x_{5} x_{4}+x_{6} x_{5} x_{2}^{2}+x_{5} x_{4} x_{2}^{3}+x_{5} x_{2}^{5},
\end{aligned}
$$

where $x_{10}=\left[P_{4}^{10}\right], x_{8}=\left[P_{4}^{8}\right], x_{9}$ is the Dold generator, $x_{6}=\left[\boldsymbol{R} P^{6}\right]$, and $x_{4}=\left[R P^{4}\right]$. Since $x_{10} x_{5}+x_{8} x_{5} x_{2}+x_{9} x_{6}+x_{9} x_{4} x_{2}$ fibers over $S^{4}$, it follows that $x_{5}^{3}+x_{6} x_{5} x_{4}+x_{6} x_{5} x_{2}^{2}+x_{5} x_{4} x_{2}^{3}+x_{5} x_{2}^{5}$ also fibers over $S^{4}$. I see no $a$ priori way of knowing this. An easy computation shows that $x_{6} x_{5} x_{4}+x_{6} x_{5} x_{2}^{2}+x_{5} x_{4} x_{2}^{3}+x_{5} x_{2}^{5}=x_{5}\left[P_{2}^{4}\right]\left[P_{2}^{6}\right]$, so we have,

Proposition 5.4. The cobordism class $x_{5}^{3}$ fibers over $S^{4}$ if and 
only if $x_{5}\left[P_{2}^{4}\right]\left[P_{2}^{5}\right]$ fibers over $S^{4}$.

6. Some results on bundles over $S^{8}$. It should be apparent that may of the tools we have developed here can also be useful in the study of bundles over $S^{8}$.

Proposition 6.1. There exist generators for $\mathfrak{R}_{*}$ which fiber over $S^{8}$ in even dimensions $\geqq 16$ and odd dimensions $\geqq 25$.

Proof. For even dimensions $\geqq 16$, we use the manifolds

$$
\left\{P_{8}^{m+8}=R P\left(H_{8}+(m-7) R\right) \mid m \in 2 Z\right\}
$$

where $H_{8}$ is the underlying real bundle of the canonical twisted Cayley line bundle over $K P^{1}=S^{8}$.

For odd dimensions, consider manifolds of the form

$$
P_{8}(m, n)=R P\left(H_{8} \times \tau P^{n}+(m-7) R\right)
$$

of dimension $m+2 n+8$. For dimensions $i=2^{p}(2 q+1)-1$, where $p>3$, the following classes are indecomposable:

$$
\left[P_{8}\left(2^{p}-9,2^{p} q\right)\right] \text {. }
$$

Since $p>3$, we must still find generators which fiber for dimensions of the form $4 q+1,8 q+3$ and $16 q+7$. These cases can be accounted for by using $P_{8}(4 q-11,2), P_{8}(8 q-13,4)$ and $P_{8}(16 q-17,8)$, although we still miss dimensions $13,17,19$ and 23 , as in the proof to 2.2.

REMARK 6.2. Recall that, according to [7, 2.3], the existence of generators which fiber over $S^{5}, S^{6}$ in $S^{7}$ in the above dimensions is also guaranteed.

REMARK 6.3. Of course there is no 23-dimensional generator which fibers, because if $F^{15} \rightarrow M^{23} \rightarrow S^{8}$ is a bundle, then

$$
s_{23}[M]=S q^{8} s_{15}[M]=v_{8} s_{15}[M]=\left\langle s_{15}, p^{*}(x) \cap[M]\right\rangle=s_{15}[F]=0 .
$$

Therefore, it is still not known whether there are generators which fiber over $S^{8}$ for dimensions 12, 13, 14, 17 and 19 .

7. Conclusion. One way of describing the problem dealt with in this paper is to pose the following question. Is there a correspondence between ideals in $\mathfrak{N}_{*}$ defined by means of algebraic conditions and those defined geometrically. Eventually, an algebraic condition should be found which neatly characterizes the geometric ideal composed of cobordism classes which fiber over $S^{k}$, but the nonexistence results 
of this paper seem to indicate that this algebraic condition will involve something other than Stiefel-Whitney numbers for large values of $k$.

\section{REFERENCES}

1. R. L. W. Brown, Cobordism and bundles over spheres, Michigan Math. J., 16 (1969), 315-320.

2. R. O. Burdick, Oriented manifolds fibered over the circle, Proc. Amer. Math. Soc., 17 (1966), 449-452.

3. P. E. Conner, The bordism class of a bundle space, Michigan Math. J., 14 (1967), 289-303.

4. P. E. Conner and E. E. Floyd, Fibering within a cobordism class, Michigan Math. J., 12 (1965), 33-47.

5. A. Dold, Erzeugende der Thomschen Algebra $\mathfrak{R}_{*}$, Math. Zeitschrift, 65 (1956), 23-35.

6. W. D. Neumann, Fibering over the circle within a bordism class, Math. Ann., 192 (1971), 191-192.

7. R. E. Stong, On fibering of cobordism classes, Trans. Amer. Math. Soc., 178 (1973), 431-447.

Received July 19, 1976

Simmons COLLEge

300 The Fenway

BOSTON, MA 02115 



\section{PACIFIC JOURNAL OF MATHEMATICS}

\section{EDITORS}

RICHARD ARENS (Managing Editor)

University of California

Los Angeles, CA 90024

R. A. BEAUMONT

University of Washington

Seattle, WA 98105

C. C. Moore

University of California

Berkeley, CA 94720

\section{J. DugundJI}

Department of Mathematics

University of Southern California

Los Angeles, CA 90007

R. Finn and J. Milgram

Stanford University

Stanford, CA 94305

\section{ASSOCIATE EDITORS}

\section{E. F. BECKENBACH}

B. H. NEUMANN

F. WoLF

K. YOSHIDA

\section{SUPPORTING INSTITUTIONS}

\author{
UNIVERSITY OF BRITISH COLUMBIA \\ CALIFORNIA INSTITUTE OF TECHNOLOGY \\ UNIVERSITY OF CALIFORNIA \\ MONTANA STATE UNIVERSITY \\ UNIVERSITY OF NEVADA \\ NEW MEXICO STATE UNIVERSITY \\ OREGON STATE UNIVERSITY \\ UNIVERSITY OF OREGON \\ OSAKA UNIVERSITY
}

\author{
UNIVERSITY OF SOUTHERN CALIFORNIA \\ STANFORD UNIVERSITY \\ UNIVERSITY OF HAWAII \\ UNIVERSITY OF TOKYO \\ UNIVERSITY OF UTAH \\ WASHINGTON STATE UNIVERSITY \\ UNIVERSITY OF WASHINGTON \\ AMERICAN MATHEMATICAL SOCIETY
}

The Supporting Institutions listed above contribute to the cost of publication of this Journal, but they are not owners or publishers and have no responsibility for its content or policies.

Mathematical papers intended for publication in the Pacific Jaurnal of Mathematics should be in typed form or offset-reproduced, (not dittoed), double spaced with large margins. Please do not use built up fractions in the text of your manuscript. You may however, use them in the displayed equations. Underline Greek letters in red, German in green, and script in blue. The first paragraph or two must be capable of being used separately as a synopsis of the entire paper. Items of the bibliography should not be cited there unless absolutely necessary, in which case they must be identified by author and Journal, rather than by item number. Manuscripts, in triplicate, may be sent to any one of the editors. Please classify according to the scheme of Math. Reviews, Index to Vol. 39. All other communications should be addressed to the managing editor, or Elaine Barth, University of California, Los Angeles, California, 90024.

The Pacific Journal of Mathematics expects the author's institution to pay page charges, and reserves the right to delay publication for nonpayment of charges in case of financial emergency

100 reprints are provided free for each article, only if page charges have been substantially paid. Additional copies may be obtained at cost in multiples of 50 .

The Pacific Journal of Mathematics is issued monthly as of January 1966. Regular subscription rate: $\$ 7200$ a year (6 Vols., 12 issues). Special rate: $\$ 36.00$ a year to individual members of supporting institutions.

Subscriptions, orders for back numbers, and changes of address should be sent to Pacific Journal of Mathematics, 103 Highland Boulevard, Berkeley, California, 94708.

PUBLISHED BY PACIFIC JOURNAL OF MATHEMATICS, A NON-PROFIT CORPORATION

Printed at Kokusai Bunken Insatsusha (International Academic Printing Co., Ltd.). 8-8, 3-chome, Takadanobaba, Shinjuku-ku, Tokyo 160, Japan.

Copyrit (C) 1975 by Pacific Journal of Mathematics Manufactured and first issued in Japan 


\section{Pacific Journal of Mathematics \\ Vol. 69, No. $2 \quad$ June, 1977}

Carol Alf and Thomas Alfonso O'Connor, Unimodality of the Lévy spectral

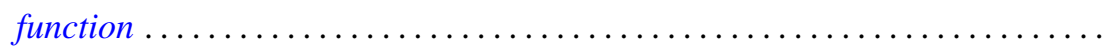

S. J. Bernau and Howard E. Lacey, Bicontractive projections and reordering of

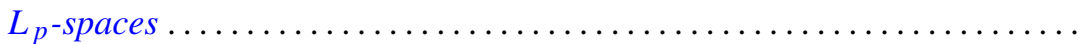

Andrew J. Berner, Products of compact spaces with bi-k and related spaces..... 303

Stephen Richard Bernfeld, The extendability and uniqueness of solutions of ordinary differential equations ...............................

Marilyn Breen, Decompositions for nonclosed planar m-convex sets ..........

Robert F. Brown, Cohomology of homomorphisms of Lie algebras and Lie

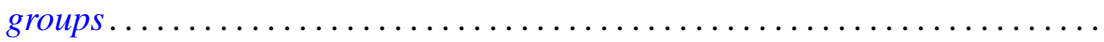

Jack Douglas Bryant and Thomas Francis McCabe, A note on Edelstein's iterative test and spaces of continuous functions ....................

Victor P. Camillo, Modules whose quotients have finite Goldie dimension ....... 333

David Downing and William A. Kirk, A generalization of Caristi's theorem with

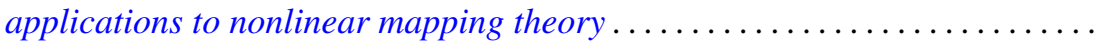

Daniel Reuven Farkas and Robert L. Snider, Noetherian fixed rings ...........

Alessandro Figà-Talamanca, Positive definite functions which vanish at

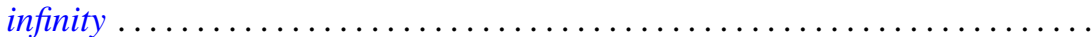

Josip Globevnik, The range of analytic extensions .................. 365

André Goldman, Mesures cylindriques, mesures vectorielles et questions de

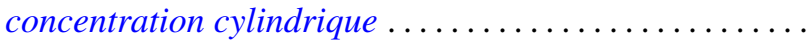

Richard Grassl, Multisectioned partitions of integers..........

Haruo Kitahara and Shinsuke Yorozu, A formula for the normal part of the

Laplace-Beltrami operator on the foliated manifold .... .

Marvin J. Kohn, Summability $R_{r}$ for double series .........

Charles Philip Lanski, Lie ideals and derivations in rings with involution ..

Solomon Leader, A topological characterization of Banach contractions . .

Daniel Francis Xavier O’Reilly, Cobordism classes of fiber bundles . .

James William Pendergrass, The Schur subgroup of the Brauer group . .

Howard Lewis Penn, Inner-outer factorization of functions whose Fourier series

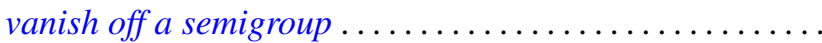

501

William T. Reid, Some results on the Floquet theory for disconjugate definite

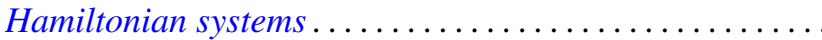

Caroll Vernon Riecke, Complementation in the lattice of convergence

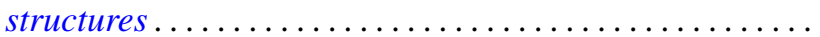

Louis Halle Rowen, Classes of rings torsion-free over their centers ......... 527

Manda Butchi Suryanarayana, A Sobolev space and a Darboux problem ....... 535

Charles Thomas Tucker, II, Riesz homomorphisms and positive linear maps.... 551

William W. Williams, Semigroups with identity on Peano continua ........... 557

Yukinobu Yajima, On spaces which have a closure-preserving cover by finite 